UDC: $811.111^{\prime} 376.625 .19 \quad$ https://doi.org/10.22190/JTESAP2104725G

\title{
MOVE-STEP STRUCTURE OF THE RESULTS AND DISCUSSION SECTION OF ELECTRONIC ENGINEERING RESEARCH ARTICLES WRITTEN BY CHINESE AND THAI RESEARCHERS
}

\author{
Sutong Gao, Issra Pramoolsook
}

Suranaree University of Technology, Thailand

\begin{abstract}
This study explored the move-step structure of the results and discussion (RD) section of electronic engineering research articles (EERAs) written by Chinese and Thai researchers. Two corpora, with each containing 12 RD sections, were compiled for analysis with reference to the three frameworks of Kanoksilapatham (2005, 2015) and Maswana et al. (2015). In addition, variations in terms of move-step structure between the two corpora were examined. Findings firstly demonstrated a newly proposed framework of 3 moves and 12 steps. What's more, all of the three moves were found obligatory, and the variations between the two corpora mainly existed in the steps under each move category. Findings of the present study could provide insights into EERA composition for novice writers. Moreover, generic variations that are acceptable within the discipline might expand research article genre knowledge for both EE researchers and genre practitioners.
\end{abstract}

Key words: move-step structure, electronic engineering research article, results and discussion section, contrastive analysis

\section{INTRODUCTION AND LITERATURE REVIEW}

Research Article (RA), one of the most important genres for producing and disseminating knowledge, has attracted extensive attention from genre scholars. Studies on RA genre generally fall into two categories: move or move-step analysis of RA structure and linguistic features that characterize the move structure. Studies of the former kind were concerned with the moves or move-step structure of RAs in a variety of fields in which move and step are the under-examined rhetorical factors contributing to the communicative purpose of a RA. A move, according to Biber et al. (2007), refers to a section of a text that performs a specific communicative function. Each move not only has its own purpose but also contributes to the overall communicative purposes of the genre. Steps are the multiple elements that together, or in some combination, realize the move (Biber et al., 2007). The studies concerning the moves or move-step structure in RA genre examined either individual sections (e.g. Basturkmen, 2012; Stoller \& Robinson, 2013; Graves et al., 2014) or the entire RA (Lin \& Evans, 2012; Kanoksilapatham, 2015; Tessuto, 2015; Kwan, 2017). On the other hand, studies of the latter

Submitted June $5^{\text {th }}, 2021$, accepted for publication August $5^{\text {th }}, 2021$

Corresponding author: Issra Pramoolsook, School of Foreign Languages, Institute of Social Technology, Suranaree University of Technology, Nakhon Ratchasima, Thailand 30000. E-mail: issra@sut.ac.th 
kind examined such linguistic features as lexical bundles (Lu \& Deng, 2019), phraseology (Le \& Harrington, 2015), and hedging (Li \& Pramoolsook, 2015). In addition, a variety of academic disciplines such as dentistry (Basturkmen, 2012), sociology (Brett, 1994), management (Lim, 2006), mathematics (Moghaddasi \& Graves, 2017), chemistry (Stoller \& Robinson, 2013), and law (Tessuto, 2015), etc. have been included in RA move and linguistic feature analyses.

The Results and the Discussion in RAs were two individual sections in most previous genre studies. As stated in Salager-Meyer (1994), the Results section is quite a straightforward unfolding of findings as it presents a clear description of the results, describes the process of manipulating the data obtained during the experimental stage, and makes limited claims about the statistical tests. Rhetorically, results convey new knowledge through presentation, explanation and interpretation of data, thus constituting the core of RA. In other words, the Results section plays a key role in demonstrating results or findings yielded from hypothesis proposed in the Introduction and the methodology employed in the Methods. It represents a carefully constructed discourse to convince readers of the validity of the scientific facts that form the basis of specific knowledge claims (Hyland, 1998).

Yang and Allison (2013) investigated the move-step structure of both the Results and the Discussion sections. Mainly investigating organizational patterning of applied linguistic empirical RAs, they found that the Results section tended to comment on results briefly and to be highly cyclical. The Discussion section, on the other hand, provided a deeper understanding of the results found, which is the unique communicative purpose of this section. The major differences in terms of communicative purposes of both the sections motivated applied linguistics researchers to use different section headings. Other studies exploring RA Results section include Brett (1994), Williams (1999), and Lim (2010). Lim (2010) adopted a mixed-method genre-based study to explore the comments in the Results section in RAs in applied linguistics and education. The study revealed disciplinary variations in terms of the four commentary steps (i) 'explaining the finding/s', (ii) 'evaluating the findings', (iii) 'comparing findings with literature', and (iv) 'making recommendations for future research'. The first three steps were prevalent in applied linguistic RAs; while 53\% of educational RAs were stripped of comments, which resembled Williams' (1999) findings concerning medical research reports.

Move-step analysis of the Discussion section has drawn more attention than the Results section (e.g. Holmes, 1997; Peacock, 2002; Basturkmen, 2012; Dujsik, 2013). The first two studies focused on investigating disciplinary variations of move structures. Holmes (1997) revealed that history texts were particularly distinctive compared with political science and sociology. Peacock's (2002) study across seven disciplines revealed interdisciplinary and NS/NNS variations in terms of the type and the number of moves and move cycles. NNS writers tended to use recognizably different discourse patterns, suggesting it challenging for NNS conforming to genre conventions that differed from those of their L1. Basturkmen (2012) examined move-step structure in dentistry research reports and revealed two patterns of argumentation in commenting moves: one centered on explanations and the other centered on comparisons and evaluations. Dujsik (2013) adopted Peacock's (2002) revised model in analyzing move-step structure of applied linguistic RAs and suggested intra-disciplinary variations within this field. Kanoksilapatham (2015) and Maswana et al. (2015) are two related studies dealing with subdisciplinary variations in terms of rhetorical structure of engineering RAs. The former 
found that the variations across three engineering subdisciplines could be interpreted in accordance with the authors' goals and the nature of the studies within the subdisciplines. The latter revealed that no common move patterns throughout the RAs were identified across the five subdisciplines due to the differences in the nature of research in each field.

In the present study, the RAs in the discipline of electronic engineering (EE) is under investigation. Electronic engineering is "a science about the devices and processes that use electromagnetic energy conversion to transfer, process, and store energy, signals and data in energy, control, and computer systems (Vodovozov, 2010, p. 8)". This cutting-edge discipline has evolved to cover many aspects of the society and is crucial for economic growth in many countries. The technologies generated from EE discipline such as the wireless communication system and video conferencing system, etc., bring magnificent benefits to people's life. However, electronic engineering research article (EERA), a key venue for engineers or engineering researchers to conduct academic communication and publish their findings, receives scant attention in genre studies. Thus, EERA becomes the focus of the present study for the purpose of revealing the move-step structures as well as the variations of EERA Results and Discussion section composed by Chinese and Thai researchers. Therefore, the present study addresses the following research questions:

1) What are the moves, steps, and move-step structures of the Results and Discussion (RD) section in electronic engineering research articles (EERAs) by Chinese $(\mathrm{CH})$ and Thai (TH) writers?

2) What are the variations of the moves, steps, and move-step structures of the Results and Discussion (RD) section in EERAs between the two groups of writers?

\section{MethodOLOGY}

To address the research questions of the current study, move-step structure of the EERAs was analyzed and compared. Being a part of a larger project that analyzes the whole EERAs, the current study focuses only on the Results and Discussion section of 24 EERAs, with 12 RD sections in both the Chinese corpus $(\mathrm{CH})$ and the Thai corpus $(\mathrm{TH})$. The selection of the journals and RAs were based on four criteria: 1) The RAs were written by Chinese and Thai authors, 2) The RAs were empirical studies in electronic engineering discipline, 3) The RAs were included in the journals indexed by SCOPUS, and 4) The RAs were published between 2019 and 2020. A disciplinary expert was invited for recommendation of the journals. For ensuring the authors' L1 status, the researchers referred to their biography, affiliations, and their bachelor degree institutions (Wood, 2001). One point worth mentioning here is that the structure of the RAs was not confined solely to the ones with clear headings of "Introduction", "Methods", "Results", and "Discussion"; namely, IMRD in short, since there were many non-IMRD RAs in the target discipline. Therefore, gathering RAs that solely conformed to the IMRD structure would certainly sabotage genuineness of data collection and limit the scope of data analysis.

Both of the two corpora were under examination for move-step structure analysis. Firstly, three frameworks (Kanoksilapatham, 2005; Kanoksilapatham, 2015; and Maswana et al., 2015) were used as references for developing a proposed coding protocol appropriate for the move-step structure of the RD sections in EERAs in the two corpora, a process that involved the researchers and an invited disciplinary expert examining $15 \%$ of the whole corpus (2 EERAs in each corpus). However, the coding protocol was subjected to subtle 
modifications in the subsequent and main coding process aiming at generating a more suitable move-step structure framework. Secondly, inter-rater reliability involving two coders working together, one being one of the researchers of the present study and the other being an invited disciplinary expert, was employed to guarantee a higher reliability of the study. Finally, this study adopted the cut-off frequency in Maswana et al. (2015), i.e., 50\%, for classifying conventional and optional moves and steps. Additionally, $80 \%$ was set as the cut-off frequency for distinguishing obligatory and conventional moves and steps. In other words, if the occurrence of one move or step is ranged from $80 \%$ to $100 \%$, it is regarded as obligatory; if in the range of $50 \%-79 \%$, it is considered as conventional. The moves and steps whose frequency of occurrence is less than $50 \%$ fall into the category of optional. The reason for setting $50 \%$ and $80 \%$ as the cut-off points for reference was that the number of EERAs in each corpus was relatively small.

Before conducting analysis, the researchers of the present study briefly examined the RD section of all the 24 EERAs in the two corpora. It was found that the majority of EERAs in the two corpora, i.e., 8 in the $\mathrm{CH}$ corpus and 7 in the TH corpus, maintained the conventional section heading "Results and Discussion". A few cases were with alternative functional headings (e.g., Results, Discussion, Experimental Validations, Implementation and Experimental Results, Experimental Results and Discussion). In addition, there was also one case arranging Results and Discussion as two separate sections (i.e., CH12). Despite the heading differences, the sections share the common characteristics of the RD section, which were reporting results and commenting on results. Therefore, all these sections were categorized as Results and Discussion (RD) in the present study for analysis.

\section{RESULTS AND DISCUSSION}

In this section, moves and steps were identified firstly to form a newly proposed framework for the EERA RD section. Then, descriptions of all of the moves and steps were provided. The status of each move and step was obtained from their frequency of occurrence. Finally, comparison of the move-step structure of the two corpora was discussed.

From the analysis, it was found that there were three moves in the RD section in the EERAs in both of the two corpora, Move 1: Preparatory information, Move 2: Reporting results, and Move 3: Commenting on results. Except for Move 2, both Move 1 and Move 3 contain six steps. The proposed framework for the RD section is shown in Table 1. The integers, 1, 2, and 3, represent the moves, and the decimals such as 1.1, 1.2, and 3.4 represent the steps of each move. 
Table 1. The proposed framework for the RD section in EERA

\begin{tabular}{|l|l|r|c|}
\hline Section & Move and Step & $\%$ & Status \\
\hline Results and & 1. Preparatory information & 91.7 & Obl \\
Discussion & 1.1 Introducing the section & 8.3 & Opt \\
$(\mathrm{N}=24)$ & 1.2 Specifying equipment or site & 8.3 & $\mathrm{Opt}$ \\
& 1.3 Explaining principles & 16.7 & $\mathrm{Opt}$ \\
& 1.4 Justifying procedures or methodology & 66.7 & $\mathrm{Con}$ \\
& 1.5 Summarizing procedures & 58.3 & $\mathrm{Con}$ \\
& 1.6 Defining terms & 8.3 & Opt \\
& 2. Reporting results & 100 & Obl \\
& 3. Commenting on results & 100 & Obl \\
& 3.1 Interpreting results & 100 & Obl \\
& 3.2 Comparing results & 16.7 & Opt \\
& 3.3 Relating to theories and previous studies & 8.3 & Opt \\
& 3.4 Summarizing results & 46 & Opt \\
& 3.5 Indicating research implications & 8.3 & Opt \\
& 3.6 Suggesting further research & 8.3 & Opt \\
\hline
\end{tabular}

Note: Con=conventional; Obl=obligatory; Opt=optional

\subsection{Description of moves and steps in the Results and Discussion section}

Move 1: Preparatory information provides background information such as principle and/or methodology of the research being reported in order to prepare for presenting the results. Functioning as a transition after the Methods section in the RD section, this move assists in presenting the results in a less abrupt way. There are six steps in this move: Introducing the section, Specifying equipment or environment, Explaining principles, Justifying procedures or methodology, Summarizing procedures, and Defining terms. Move 1 can be accomplished when there is at least one of the six steps occurring in this section. According to the cut-off occurrence rate in this study ( $\geqslant 80 \%$ : obligatory; 50\%-79\%: conventional; <50\%: optional), Move 1 Preparatory information is an obligatory move in the two corpora.

Step 1.1: Introducing the section is present at the very beginning of the section. By providing an overview of the section through reemphasizing the purposes or methods, it functions as a brief introduction to the section. Only 2 EERAs in the TH corpus employed this step, suggesting that it is optional. An example of Step 1.1 is provided below.

(1) In this section, the result of the DTTV-SFN propagation with the proposed measurement model will be discussed. The measured results will be evaluated by power delay profile, path loss, the comparison between spectrum variation and delay, CNR and delay, modulation error ratio and delay, and noise margin and delay. (TH12)

Step 1.2: Specifying equipment or site occurs when writers need to give specific details about the devices, apparatuses, or equipment involved in the experiment and the site or environment in which the research or experiment took place. This is a step employed especially in studies that stress the role of the equipment, device, or site, since they are part of methodology that have a certain impact on the results or findings of the study. The low frequency of occurrence of this step indicated that this optional step occurred in a few EERAs in the two corpora. Example 2 below demonstrates Step 1.2. 
In order to verify the communication performances of the designed RFID sensor, this paper adopts a special RFID tester of VISN-R1200 from JX Instrumentation, China. The test environment is shown in Fig. 7. The Bosch VCL4003 climate box is used to test the temperature and humidity performances of the proposed wireless sensor. The RFID sensor tag designed in this paper is fabricated with discrete components. The base material of the sensor is FR4 and it covers the area of $12 \times 8 \mathrm{~cm} 2$. (CH3)

Step 1.3: Explaining principles is another optional step of Move 1. The laws, rules or theories that the research is based on would be illustrated or explained before reporting the relevant results, a process that lays foundation and convinces the readers of reasonableness of the experimental methodology and the validity of the results. The occurrence of this step was only 12 in the whole corpus. To illustrate the function of Step 1.3, one excerpt is chosen as an example below.

(3) The inline successive pumping scheme operates when a fraction of the power is coupled into the panda ring resonator. The $E_{\text {in }}$ value of the panda ring arises from that fraction of the power and circulates in the optical system. Ultimately the resonant output is achieved at the throughput port, at the inline MZI. (TH3)

Step 1.4: Justifying procedures or methodology occurs much more frequently in the two corpora than the three previous steps. This step is achieved through demonstrating to the readers the rationale of selecting a certain experimental method or the reason of conducting the research by adopting a series of processes. In addition, researchers in this discipline tend to adopt mathematical algorithm such as equations to prove their rationality of relevant process. The total occurrence of Step 1.4 was 84, the second highest step among all the steps of Move 1. Twenty-six EERAs demonstrated this step. Example 4 below illustrates Step 1.4.

(4) Several tower waveguide structures are printed with different dimensions to determine the optimum parameters needed to ensure the highestconfinementwithin the guiding region. (TH2)

Step 1.5: Summarizing procedures is another frequent step in the EERAs in the two corpora. In this step, writers describe a process or a series of actions involved in a particular work or an experiment intending to achieve a result. This step sometimes follows Step 1.4 Justifying procedures or methodology. In this case, the process seems more appropriate and convincing to the reviewers and readers when there are sounding reasons provided. Example 5 is for the demonstration of Step 1.5.

(5) In this case, the transmitter was placed in front of the second car and kept $3 \mathrm{~m}$ from the train door. The receiver was moved inside the train along the corridor of the first two cars. Then the operator moved outside and acquired the reference power level near the door and then re-entered the train. These measurements were repeated at both $2.4 \mathrm{GHz}$ and $5.7 \mathrm{GHz}$. (CH8)

Step 1.6: Defining terms is one of the optional steps in Move 1. It has the least frequency of occurrence in the two corpora. It provides the definition of technical terms that the writers assume necessary and worthy of explanation. To illustrate the function of Step 1.6, Example 6 is given below.

(6) The return loss characteristic $\left(S_{I I}\right)$ refers to the intensity of electromagnetic wave reflected when the electromagnetic wave is transmitted through the antenna. $(\mathrm{CH} 3)$

Move 2: Reporting results is the dominant move, with $100 \%$ occurrence frequency, in the RD section in the two corpora. This move is to demonstrate or depict results and 
findings, usually in the form of statistics. In the EERAs of the whole corpus, the obligatory status of Move 2 is based on the results and findings of every stage of the testing of a model or an experiment, thus maintaining the second dominant position among all the moves and steps. Example 7 demonstrates Move 2 as shown below.

(7) In Table 2, the comparison of delay characteristic in each positions are presented. The received signal time delay from station 1 are ranging from 20.23 to $54.52 \mu$ s and the average time delay is $38.94 \mu \mathrm{s}$. (TH12)

The purpose of Move 3: Commenting on results has the purpose to provide or establish the meaning from the statistical results reported and the examples demonstrated in Move 2. This move includes information and interpretations that go beyond the "objective" results (Yang \& Allison, 2003). This may engage the various ways to interpret the results in the context of the study, to illustrate the contribution of the findings to the field (usually in comparison with relevant literature), the potential or underlying causes of the results, or comments on the strength, limitations of the results (Yang \& Allison, 2003). Specifically, this move could be realized through the following steps: Step 3.1 Interpreting results, Step 3.2 Comparing results, Step 3.3 Relating to theories and previous studies, Step 3.4 Summarizing results, Step 3.5 Indicating research implications and Step 3.6 Suggesting further research.

Step 3.1: Interpreting results is the most frequent step that appears within Move 3 Commenting on results. All the 36 EERAs in the two corpora contain this step owing to the fact that electronic engineering researchers tend to provide reasons and explanations for the results yielded in each stage of the study. This is different from Yang and Allison's (2003) findings on the Results section. They found that Interpreting results, which was Step 1 of Move 3 Commenting on results, ranked the second after Step 2 Comparing results with literature. Disciplinary disparity might be one of the reasons that contribute to the difference of the finding. Example 8 below illustrates the function of Step 3.1.

The sensing signal increased almost 13 times using our proposed antenna array for lactose detection. These results showed that the sensing performance of this structure working at reflection mode was even better than that at transmission mode. This was mainly due to the fact that the resonance in reflection mode had a higher quality factor and larger local field enhancement. (CH1)

Step 3.2: Comparing results is another form of commenting on results. The current results and findings are compared with those in the previous studies in the literature, which also shows the writer's own understanding of the connection between the previous and current research. Usually, the phrases such as "comparing...with...", "compared with...", "be consistent with..." are the obvious linguistic clues for this step. In addition, in-text citation might also suggest this step. The relatively low frequency of occurrence suggests that this step is optional. Example 9 below illustrates the function of Step 3.2.

(9) Comparing the probe performance with the previous works, the calibration factor of a LTTC probe is about $60 \mathrm{dBS} / \mathrm{m}$ which is higher than the proposed probe since the LTTC magnetic pick- up area is smaller but the smaller pick-up area has better magnetic field spatial resolution. (TH9) 
Step 3.3: Relating to theories and previous studies is a step where the writers introduce previous relevant studies in terms of their methodology, results, and findings, which would be commented on or adopted for the current research. The reason for EE researchers using this step is that they might need theories, methods, or results of the previous studies to back up their current study by either indicating their strengths or drawbacks. Thus, the persuasion seems more convincing to the reviewers and readers. In-text citation is an apparent indication for this step. Example 10 is an illustration of this step.

(10) Traditional soil sampling method (Hedayati-Dezfooli and Leong, 2019) shows high accuracy performance, but it is time-consuming and laborious, and is not suitable for real-time monitoring. The soil environmental monitoring system based on wired communication (Zhang et al., 2015) exhibits the advantages of huge data transmission and fast transmission speed, but its deployment process is complex and maintenance cost is high. (CH3)

Step 3.4: Summarizing results provides a short statement regarding the main points of the results and findings. It functions as a small conclusion of the present research or part of research. This step usually appears at the end of the RD section. Example 11 below is the illustration for Step 3.4.

(11) With these characteristics, the proposed antenna is acceptable for use in indoor base stations with triple-frequency bands, directional radiation patterns, high gain, and high power handling. (TH7)

Step 3.5: Indicating research implications offers possible effect or practicability deducted from the results or findings of the research being reported. Electronic engineering is a discipline that has close relation with people's daily lives, therefore, some EE researchers apply this step to indicate that their proposed model or method is beneficial for resolving certain problems existing in the field or in people's lives. Example 12 is the illustration for Step 3.5.

(12) An especially LiFi network is most beneficial for radiology room. The ad hoc accessed network can also be linked to the long-haul transmission via the internet of thing (IoT) for more required applications. (TH11)

Step 3.6: Suggesting further research is a step for researchers to recommend a theme or a direction for future research relevant to current study. It is regarded as optional since only one EERA was found to have this step. Example 13 below illustrates this step.

(13) The extra loss due to the windows may be different for different trains and different environments in which the train is operated, hence inducing a different amount of wave reentering. However, these are topics for future research. $(\mathrm{CH} 8)$

\subsection{Comparison of the move-step structures}

\subsubsection{Move and step}

Tables 2-3 summarize similarities and variations between the $\mathrm{CH}$ and the $\mathrm{TH}$ corpora in the frequency of occurrence of Moves 1-3. Move 1: Preparatory information occurred in $100 \%$ of the EEERAs in the $\mathrm{CH}$ (Average occurrence: 4.25 per section) and in $83.3 \%$ of the EERAs in the TH (Average occurrence: 2.58 per section). Move 2: Reporting results occurred $100 \%$ in both of the two corpora, but with the average occurrence per section of 5.83 and 3.33 in the $\mathrm{CH}$ and the $\mathrm{TH}$, respectively. Move 3: Commenting on results, similar to Move 2, also occurred in all of the EERAs in both the $\mathrm{CH}$ and the $\mathrm{TH}$, with the average occurrence per section of 5.92 and 3.33, respectively. Since Moves 1, 2 and 3 occurred in more than $80 \%$ of the both corpora, they were all obligatory. The apparent variations were 
found in the two steps under Move 1 and Move 3, which were Step 1.4 Justifying procedures or methodology, and Step 3.4 Summarizing results. Step 1.4 was regarded as an obligatory step in the $\mathrm{CH}$ corpus; while it was a conventional step in the TH corpus. Step 3.4 Summarizing results in the $\mathrm{CH}$ was viewed as an optional step; however, it was considered conventional in the TH. The other steps under Move 1 and Move 3 in the two corpora demonstrated similarities rather than variations. That is, the same step fell into the same category in the two corpora.

Table 2. The number of EERAs in which move or step occurs in the $\mathrm{CH}$ and the $\mathrm{TH}$

\begin{tabular}{|l|r|r|r|r|r|r|}
\hline \multirow{2}{*}{ Move/Step } & \multicolumn{2}{c|}{$\begin{array}{c}\mathrm{CH} \\
\text { (RA=12) }\end{array}$} & \multicolumn{2}{c|}{$\begin{array}{c}\mathrm{TH} \\
\text { (RA=12) }\end{array}$} & \multicolumn{2}{c|}{$\begin{array}{c}\text { Whole corpus } \\
\text { (RA=24) }\end{array}$} \\
\cline { 2 - 7 } & $\begin{array}{c}\text { No. of } \\
\text { RAs }\end{array}$ & $\%$ & $\begin{array}{r}\text { No. of } \\
\text { RAs }\end{array}$ & $\%$ & $\begin{array}{r}\text { No. of } \\
\text { RAs }\end{array}$ & $\%$ \\
\hline 1. Preparatory information & 12 & 100.0 & 10 & 83.3 & 22 & 91.7 \\
1.1 Introducing the section & 0 & 0.0 & 1 & 8.3 & 1 & 8.3 \\
1.2 Specifying equipment or & 1 & 8.3 & 0 & 0.0 & 1 & 8.3 \\
$\quad$ environment & & & & & & \\
1.3 Explaining principles & 3 & 25.0 & 1 & 8.3 & 4 & 16.7 \\
1.4 Justifying procedures or & 10 & 83.3 & 6 & 50.0 & 16 & 66.7 \\
$\quad$ methodology & 7 & 58.3 & 7 & 58.3 & 14 & 58.3 \\
1.5 Summarizing procedures & 1 & 8.3 & 0 & 0.0 & 1 & 8.3 \\
1.6 Defining terms & 12 & 100.0 & 12 & 100.0 & 24 & 100.0 \\
2. Reporting results & 12 & 100.0 & 12 & 100.0 & 24 & 100.0 \\
3. Commenting on results & 12 & 100.0 & 12 & 100.0 & 24 & 100.0. \\
3.1 Interpreting results & 1 & 8.3 & 3 & 25.0 & 4 & 16.7 \\
3.2 Comparing results & 1 & 8.3 & 1 & 8.3 & 2 & 8.3 \\
3.3 Relating to theories and & & & & & & \\
$\quad$ previous studies & 5 & 41.7 & 6 & 50.0 & 11 & 46.0 \\
3.4 Summarizing results & 0 & 0.0 & 1 & 8.3 & 1 & 8.3 \\
3.5 Indicating research implications & 1 & 8.3 & 0 & 0.0 & 1 & 8.3 \\
3.6 Suggesting further research & & & & & & \\
\hline
\end{tabular}


Table 3. The occurrence of move or step in the $\mathrm{CH}$ and the $\mathrm{TH}$

\begin{tabular}{|l|r|r|r|r|}
\hline & \multicolumn{2}{|c|}{$\mathrm{CH}(\mathrm{RA}=12)$} & \multicolumn{2}{c|}{ TH (RA=12) } \\
\hline Move/Step & Occurrence & $\begin{array}{c}\text { Average } \\
\text { Occurrence } \\
\text { per section }\end{array}$ & Occurrence & $\begin{array}{c}\text { Average } \\
\text { Occurrence } \\
\text { per section }\end{array}$ \\
\hline 1. Preparatory information & 51 & 4.25 & 31 & 2.58 \\
1.1 Introducing the section & 0 & 0.00 & 1 & 0.08 \\
1.2 Specifying equipment or & 2 & 0.17 & 0 & 0.00 \\
$\quad$ environment & 4 & 0.33 & 1 & 0.08 \\
1.3 Explaining principles & 38 & 3.17 & 16 & 1.33 \\
1.4 Justifying procedures or & 16 & 1.33 & 18 & 1.50 \\
$\quad$ methodology & 4 & 0.33 & 0 & 0.00 \\
1.5 Summarizing procedures & 70 & 5.83 & 40 & 3.33 \\
1.6 Defining terms & 71 & 5.92 & 40 & 3.33 \\
2. Reporting results & 69 & 5.75 & 34 & 2.83 \\
3. Commenting on results & 1 & 0.08 & 3 & 0.25 \\
3.1 Interpreting results & 1 & 0.08 & 1 & 0.08 \\
3.2 Comparing results & & & & \\
3.3 Relating to theories and & 5 & 0.42 & 7 & 0.58 \\
$\quad$ previous studies & 0 & 0.00 & 1 & 0.08 \\
3.4 Summarizing results & 1 & 0.08 & 0 & 0.00 \\
3.5 Indicating research implications & 333 & & 193 & \\
3.6 Suggesting further research & & & & \\
\hline Total & & & & \\
\hline
\end{tabular}

The variations identified among the two corpora were Step 1.4 Justifying procedures and Step 3.4 Summarizing results based on Table 4. Only half of the corpus i.e. 6 EERAs employed this step in the TH corpus. Usually, this step in the two corpora was accompanied by Step 1.5 Summarizing procedures. The writers in EE discipline tended to firstly provide rationales or reasons for the following procedures so that they could sound reasonable. This is one characteristic of this discipline that every step of the experiment process should be based on some reasons such as mathematical algorithm or well-founded setup for testing or experiment. Ten EERAs in the $\mathrm{CH}$ corpus demonstrated this step. According to Table 3, the total number of moves and steps in the TH corpus differed significantly from that of $\mathrm{CH}$ corpus. That explained, to a certain degree, the reason for Step 1.4 missing in some EERAs in the TH. Firstly, it was the length of the whole RAs that influences the adoption of certain moves and steps. Secondly, Step 1.4 Justifying procedures or methodology appearing less frequently than Step 1.5 Summarizing procedures in the RD section in the TH could be attributed to the reason that it had already appeared in the Methods section. Thus, considering redundancy for the RD section, this step could be omitted.

Step 3.4 Summarizing results appeared in 5 and 6 EERAs in the $\mathrm{CH}$ and the $\mathrm{TH}$, respectively, which did not show great difference. However, according to the cut-off point, this step in the $\mathrm{CH}$ was considered optional, while it was regarded as conventional in the $\mathrm{TH}$. In the $\mathrm{CH}$, this step occurred more frequently than the other optional steps, suggesting that a summary or short statements for the results and findings discovered could serve as a more prevalent step. It was observed that there were many results yielded from different steps of one experiment or even different experiments or tests in EERAs, which indicated 
that this step was necessary for those researchers who synthesized the results. Other differences were not noticeable between the two corpora.

Table 4 The differences of obligatory, conventional and optional moves and steps between the $\mathrm{CH}$ and the $\mathrm{TH}$

\begin{tabular}{|c|c|c|}
\hline Move/Step & $\mathrm{CH}$ & $\mathrm{TH}$ \\
\hline $\begin{array}{l}\text { Obligatory } \\
(\geqslant 80 \%)\end{array}$ & \begin{tabular}{|l|} 
1. Preparatory information \\
1.4 Justifying procedures or \\
methodology \\
2. Reporting results \\
3. Commenting on results \\
3.1 Interpreting results \\
\end{tabular} & $\begin{array}{l}\text { 1. Preparatory information } \\
\text { 2. Reporting results } \\
\text { 3. Commenting on results } \\
\text { 3.1 Interpreting results }\end{array}$ \\
\hline $\begin{array}{l}\text { Conventional } \\
(50 \%-79 \%)\end{array}$ & & $\begin{array}{ll}1.4 & \begin{array}{l}\text { Justifying procedures or } \\
\text { methodology }\end{array} \\
3.4 & \text { Summarizing results } \\
\end{array}$ \\
\hline $\begin{array}{l}\text { Optional } \\
(<50 \%)\end{array}$ & 3.4 Summarizing results & \\
\hline
\end{tabular}

\subsubsection{Move cycle}

As indicated by the frequency of the moves and steps, the RD section is highly cyclical, one of the common characteristics of all EERAs in the two corpora. As can be seen from Tables 5-6, in total, 83 move cycles falling into 9 types were identified in the two corpora. The average number of move cycles per article was 4.5 in the $\mathrm{CH}$, and 2.4 in the TH. Not all the 9 types of move cycles appeared in both the corpora. That is, 8 and 7 types of move cycles were found in the $\mathrm{CH}$ and the $\mathrm{TH}$, respectively.

Move cycles including 2-3.1 (2 Reporting Results-3.1 Interpreting results) and 1.4-2-3.1 (1.4 Justifying procedures or methodology-2 Reporting Results-3.1 Interpreting results) demonstrated similarity between the two corpora for being the top 2 cycles in each corpus. The move cycle 2-3.1 was the most dominant one, which occurred in all the 12 RAs, accounting for $46.3 \%$ of all the move cycles in the $\mathrm{CH}$. Even though this cycle occurred in fewer RAs in the TH than those in the $\mathrm{CH}$, it remained in the highest number of the RAs in the TH. The occurrence percentage of move cycle 2-3.1 in the TH corpus accounted for $55.2 \%$ of all the nine move cycles, even higher than that in the $\mathrm{CH}$. The high percentage and occurrence indicated that this move cycle was conventional in both of the two corpora. The move cycle 1.4-2-3.1 was another frequent cycle in the two corpora, indicating that Step 1.4 was the most frequent step happening before the move cycle 2-3.1. Move cycles occurring less frequently in the two corpora were 1.5-3.1, 3.1-3.4, 1.5-2-3.1, 2-3.1-3.4 and 1.4-1.5, with the occurrence in less than 2 RAs per corpus. It was found that the move cycles in the RD section appeared to be complex since each cycle often comprised a combination of moves and steps. One move cycle could appear twice or more in one paragraph. 
Table 5. The number of EERAs in which move cycles occur in the $\mathrm{CH}$ and the $\mathrm{TH}$

\begin{tabular}{|l|c|c|c|c|}
\hline \multirow{2}{*}{ Move cycle } & \multicolumn{2}{|c|}{$\mathrm{CH}(\mathrm{RA}=12)$} & \multicolumn{2}{c|}{ TH (RA=12) } \\
\cline { 2 - 5 } & No. of RAs & $\%$ & No. of RAs & $\%$ \\
\hline $2-3.1$ & 12 & 100.0 & 8 & 66.7 \\
$1.4-2-3.1$ & 5 & 41.7 & 3 & 25.0 \\
$3.1-3.4$ & 2 & 16.7 & 2 & 16.7 \\
$1.5-2-3.1$ & 1 & 8.3 & 2 & 16.7 \\
$1.4-1.5$ & 1 & 8.3 & 2 & 16.7 \\
$2-3.1-2-3.1$ & 4 & 33.3 & 1 & 8.3 \\
$1.4-3.1$ & 4 & 33.3 & 0 & 0.0 \\
$1.5-3.1$ & 2 & 16.7 & 0 & 0.0 \\
$2-3.1-3.4$ & 0 & 0.0 & 2 & 16.7 \\
\hline
\end{tabular}

Table 6 The occurrence of move cycles in the $\mathrm{CH}$ and the $\mathrm{TH}$

\begin{tabular}{|l|rrrrrr|}
\hline & \multicolumn{3}{|c}{$\mathrm{CH}(\mathrm{RA}=12)$} & \multicolumn{2}{c|}{$\mathrm{TH}(\mathrm{RA}=12)$} & \multicolumn{2}{c|}{ Whole corpus (RA=24) } \\
\cline { 2 - 7 } Move cycle & Occurrence & \multicolumn{1}{c}{$\%$} & Occurrence & $\%$ & Occurrence & $\%$ \\
\hline $2-3.1$ & 25 & 46.3 & 16 & 55.2 & 41 & 49.4 \\
$1.4-2-3.1$ & 10 & 18.5 & 4 & 13.8 & 14 & 16.9 \\
$3.1-3.4$ & 2 & 3.7 & 2 & 6.9 & 4 & 4.8 \\
$1.5-2-3.1$ & 2 & 3.7 & 2 & 6.9 & 4 & 4.8 \\
$1.4-1.5$ & 2 & 3.7 & 2 & 6.9 & 4 & 4.8 \\
$2-3.1-2-3.1$ & 6 & 11.1 & 1 & 3.5 & 7 & 8.4 \\
$1.4-3.1$ & 5 & 9.3 & 0 & 0.0 & 5 & 6.0 \\
$1.5-3.1$ & 2 & 3.7 & 0 & 0.0 & 2 & 2.4 \\
$2-3.1-3.4$ & 0 & 0.0 & 2 & 6.9 & 2 & 2.4 \\
\hline Total & 54 & 100.0 & 29 & 100.0 & 83 & 100.0 \\
Average & 4.5 & & 2.4 & & 3.5 & \\
\hline
\end{tabular}

The obvious difference of move cycle between the two corpora was the total number of move cycles identified and the number of occurrence of certain move cycles. EERAs in the $\mathrm{CH}$ demonstrated 54 occurrence of move cycle and 29 were identified in the TH. Chinese researchers tended to employ more move cycles than Thai researchers, and this might be due to their research methodology requiring more results to be reported and commented on. A delicate difference was the occurrence of the move cycles 2-3.1-2-3.1 and 1.4-3.1. They both appeared in 4 RAs in the $\mathrm{CH}$. However, the move cycle 2-3.1-2-3.1 occurred in one RA in the TH. The move cycle 1.4-3.1 did not occur at all in the TH. The reason why 1.4-3.1 occurred in 4 RAs, i.e., 5 times in the $\mathrm{CH}$, could be that EE researchers referred to the results by pointing to the visuals inserted in the RAs, making possible that Move 3 is directly followed by Move 1. For instance:

(24) Since 12 measuring electrodes are arranged on the cutterhead, according to the measuring method described in Section 2.1, 11 voltage data can be obtained by supplying power to one measuring electrode at a time, and 132 voltage data can be obtained by supplying power to these 12 measuring electrodes in turn. (Step 1.4) As can be seen from Figures 5 and 6 , when the same amount of current is supplied to the exciting electrode and the guard electrode, the farther away the low resistivity anomalous body is from the cutterhead, the greater the voltage measured by each measuring electrode. (Step 3.1) (CH4) 
The findings suggested the move cycle 2-3.1 was the core in both of the two corpora since most move cycles identified revolved around it such as the move cycles 1.4-2-3.1, 1.5-2-3.1 and 2-3.1-2-3.1. The findings also revealed that the most frequent step that happened before the move cycle 2-3.1 was Step 1.4, while the most common step appearing after it was Step 3.4 and the move cycle itself: 2-3.1. This is consistent with Yang and Allison (2003) in that the sequence of moves and steps in each cycle follows the order shown in their proposed framework, that is, if Move 1 is absent, then Move 2 is the initial element in a cycle, followed by Move 3.

\section{CONCLUSION}

The findings of the present study suggest a move-step structure framework containing 3 moves and 12 steps for electronic engineering research articles. All the three moves were categorized as obligatory. The variations between the two groups were mainly demonstrated by steps within each move. For instance, Step 1.4 Justifying procedures or methodology was considered as obligatory in the $\mathrm{CH}$, while it was optional in the $\mathrm{TH}$. In addition, Move cycles 2-3.1 and 1.4-2-3.1, with the highest occurrence, demonstrated similarity rather than variation between the two corpora. The obvious difference in terms of move cycle was the total number of move cycles identified and the frequency of occurrence of certain move cycles. The probable reasons for Chinese researchers employing more move cycles attributed to the research purposes, scope, and methodology. For example, the methodology they adopted consisted of more than one procedures, and each procedure yielded different results that influenced the following procedure. Thus, these different procedures might be identified as different move cycles.

Since the present study is a part of a major project, which aims to investigate the move-step structure for the whole EERAs, it could firstly contribute to addressing the research questions and building an overall and a comprehensive move-step structure of EERA. Secondly, the findings of the present study could provide a writing guideline for electronic engineering researchers and students in composing the RD section of their RAs. Thirdly, the variations in terms of the moves, steps, and move cycles between these two writer groups, given that all the EERAs were successfully published, could serve as two references for ESP or EAP instructors to design academic writing courses, syllabus, and teaching materials.

However, the limitations of the present study also exist, which mainly lie in the data collection process since a few factors, including the number of words of each EERA, the unequal number of each journal that the EERAs come from, the impact factor and the Quartile in Category of the journals, were left out of concern. First, the researchers of the present study were aware of the fact that the word count of each EERA might have certain impact on the move-step structure, but they might not be the determining factors influencing the features under examination. The second limitation was the unequal number of each journal that the EERAs came from. Previously the researchers decided to collect an equal number of EERA from an equal number of the same journals. Nevertheless, this seemed an impossible mission due to the scope of the study covering two different groups of writers, which resulted in tiny intersection or overlap that was interpreted as a failed attempt experienced by the invited disciplinary insider and the researchers during data collection. Finally, the Impact Factor and the Quartile in Category of target journals were not included as the criteria of selection of journals because SCOPUS is a database famous 
for its trustworthiness of journal quality. Since both factors were capable of enhancing validity of the study, it is suggested that both or either one of them could be taken into consideration in further research in the future.

\section{REFERENCES}

Basturkmen, Helen. 2012. A genre-based investigation of discussion sections of research articles in dentistry and disciplinary variation. Journal of English for Academic Purposes 11: 134-144. https://doi.org/10.1016/j.jeap.2011.10.004

Biber, Douglas, Ulla Connor, and Thomas Upton. 2007. Discourse on the Move: Using Corpus Analysis to Describe Discourse Structure. John Benjamins Publishing.

Brett, Paul. 1994. A genre analysis of the results section of sociology articles. English for Specific Purposes 13 (1): 47-59. https://doi.org/10.1016/0889-4906(94)90024-8

Dujsik, Darunee. 2013. A genre analysis of research article discussions in applied linguistics. Language Research 49 (2):453-477.

Graves, Heather, Shahin Moghaddasi, and Azirah Hashim. 2014 "Let G 1/4 (V, E) be a graph": Turning the abstract into the tangible in introductions in mathematics RAs. English for Specific Purposes 36: 1-11. https://doi.org/10.1016/j.esp.2014.03.004

Holmes, Richard. 1997. Genre analysis, and the social sciences: An investigation of the structure of research article discussion sections in three disciplines. English for Specific Purposes 16 (4): 321-337. https://doi.org/10.1016/S0889-4906(96)00038-5

Hyland, Ken. 1998. Hedging in Scientific Research Articles. John Benjamins Publishing Company.

Kanoksilapatham, Budsaba. 2005. Rhetorical structure of biochemistry RAs. English for Specific Purposes 24: 269-292. http://dx.doi.org/10.1016/j.esp.2004.08.003

Kanoksilapatham, Budsaba. 2015. Distinguishing textual features characterizing structural variation in RAs across three engineering sub-discipline corpora. English for Specific Purposes 37: 74-86. https://doi.org/10.1016/j.esp.2014.06.008

Kwan, Becky S.C. 2017. A cross-paradigm macro-structure analysis of research articles in Information Systems. English for Specific Purposes 45: 14-30. https://doi.org/10.1016 /j.esp.2016.08.002

Le, Thi Ngoc Phuong, and Michael Harrington. 2015. Phraseology used to comment on results in the discussion section of applied linguistics quantitative research articles. English for Specific Purposes 39: 45-61. https://doi.org/10.1016/j.esp.2015.03.003

Li, Qian, and Issra Pramoolsook. 2015. Research article abstracts in two subdisciplines of Business--move structure and hedging between Management and Marketing. English Language Teaching 8 (1): 52-62. https://doi.org/10.5539/elt.v8n1p52

Lim, Jason Miin Hwa. 2006. Method sections of management research articles: A pedagogically motivated qualitative study. English for Specific Purposes 25: 282-309. https://doi.org/10.1016/j.esp.2005.07.001

Lim, Jason Miin Hwa. 2010. Commenting on research results in applied linguistics and education: A comparative genre-based investigation. Journal of English for Academic Purposes 9 (4): 280-294. https://doi.org/10.1016/j.jeap.2010.10.001

Lin, Ling, and Stephen Evans. 2012. Structural patterns in empirical research articles: A cross-disciplinary study. English for Specific Purposes 31: 150-160. https://doi.org/10. 1016/j.esp.2011.10.002 
Lu, Xiaofei, and Jinlei Deng. 2019. With the rapid development: A contrastive analysis of lexical bundles in dissertation abstracts by Chinese and L1 English doctoral students. Journal of English for Academic Purposes 39: 21-36. https://doi.org/10.1016/ j.jeap.2019.03.008

Maswana, Sayako, Toshiyuki Kanamaru, and Akira Tajino. 2015. Move analysis of research articles across five engineering fields: What they share and what they do not. Ampersand 2: 1-11. https://doi.org/10.1016/j.amper.2014.12.002

Moghaddasi, Shahin, and Heather A.B. Graves. 2017. "Since Hadwiger's conjection... is still open": Establishing a niche for research in discrete mathematics research article introductions. English for Specific Purposes 45: 69-85. https://doi.org/10.1016/j.esp. 2016.09.003

Peacock, Matthew. 2002. Communicative moves in the discussion section of research articles. System 30 (4): 479-497. https://doi.org/10.1016/S0346-251X(02)00050-7

Salager-Meyer, Françoise. 1994. Hedges and textual communicative function in medical English written discourse. English for Specific Purposes 13 (2): 149-170. https://doi.org/10. 1016/0889-4906(94)90013-2

Stoller, Fredricka L., and Marin S. Robinson. 2013. Chemistry journal articles: An interdisciplinary approach to move analysis with pedagogical aims. English for Specific Purposes 32: 45-57. https://doi.org/10.1016/j.esp.2012.09.001

Tessuto, Girolamo. 2015. Generic structure and rhetorical moves in English-language empirical law research articles: Sites of interdisciplinary and interdiscursive cross-over. English for Specific Purposes 37: 13-26. https://doi.org/10.1016/j.esp. 2014.06.002

Vodovozov, Valery. 2010. Introduction to Electronic Engineering. Bookboon.com. https://bookboon.com/en/introduction-to-electronic-engineering-ebook (accessed February 26, 2021).

Williams, Ian. A. 1999. Results sections of medical research articles: Analysis of rhetorical categories for pedagogical purposes. English for Specific Purposes 18 (4): 347-366. https://doi.org/10.1016/S0889-4906(98)00003-9

Wood, Alistair. 2001. International scientific English: The language of research scientists around the world. In Research Perspectives on English for Academic Purposes, edited by J. Flowerdew and M. Peacock, 71-83. Cambridge University Press. https://doi.org/ 10.1017/CBO9781139524766.008

Yang, Ruiying, and Desmond Allison. 2003. Research articles in applied linguistics: moving from results to conclusions. English for Specific Purposes 22: 365-385. https://doi.org/10.1016/S0889-4906(02)00026-1 